\title{
Púrpura trombocitopênica trombótica: o papel do fator von Willebrand e da ADAMTS13
}

\author{
Thrombotic thrombocytopenic purpura: the role of von Willebrand factor and ADAMTS13
}

Leandro C. Tonaco ${ }^{1}$

Danyelle R. A. Rios ${ }^{2}$

Lauro M. Vieira ${ }^{3}$

Maria das Graças Carvalho

Luci M. S. Dusse

\begin{abstract}
A púrpura trombocitopênica trombótica (PTT) instala-se de modo abrupto e é caracterizada pela oclusão difusa de arteríolas e capilares da microcirculação, levando à isquemia de tecidos. A oclusão é causada por microtrombos compostos basicamente de plaquetas e fator von Willebrand $(F v W)$. O FvW é uma glicoproteina de estrutura multimérica sintetizada exclusivamente por células endoteliais e megacariócitos. Este fator promove a adesão das plaquetas ao endotélio lesado, participa do processo de agregação plaquetária e é a proteína carreadora do fator VIII na circulação. Em condições fisiológicas, os grandes multímeros do FvW encontram-se dentro das células endoteliais e nas plaquetas e não estão presentes no plasma. Tão logo estes grandes multímeros são liberados da célula endotelial, são clivados e removidos da circulação pela enzima ADAMTS13 (A Desintegrin and Metalloprotease with eight Thrombo Spondin-1-like). A deficiencia funcional ou quantitativa de ADAMTS13 resulta no acúmulo de grandes multímeros de $F v W$ no plasma, propiciando a agregação das plaquetas e oclusão difusa das arteríolas e capilares. A maioria dos casos de PTT está associada à deficiência da ADAMTS13 e já estão disponíveis no mercado internacional conjuntos diagnósticos para a determinação dos níveis de antígenos desta enzima, da de sua atividade e dos anticorpos anti-ADAMTS13. A avaliação laboratorial da ADAMTS13 parece constituir um avanço para o diagnóstico precoce da PTT. No entanto, a interpretação dos resultados exige cautela e um conhecimento do princípio do método, bem como das etapas das reações envolvidas. Rev. Bras. Hematol. Hemoter. 2010;32(2):155-161.
\end{abstract}

Palavras-chave: Púrpura trombocitopênica trombótica; Fator von Willebrand; agregação plaquetária.

\section{Púrpura trombocitopênica trombótica (PTT)}

A púrpura trombocitopênica trombótica (PTT) foi descrita em 1924 pelo pesquisador Moschowitz e é referida também como síndrome de Moschowitz. APTT constitui uma doença rara (5-10 casos/1.000.000 de pessoas/ano), que afeta ambos os sexos, sendo sua incidência duas a três vezes maior em mulheres entre 30 e 40 anos de idade. ${ }^{1}$ A PTT pode ser hereditária ou adquirida, instala-se de modo abrupto e é caracterizada pela oclusão difusa de arteríolas e capilares da microcirculação, levando à isquemia de tecidos. A oclusão é causada por microtrombos compostos basicamente de plaquetas, formados após agregação plaquetária intravascular. Este fenômeno resulta em um quadro de anemia hemolítica

\footnotetext{
${ }^{1}$ Farmacêutico.

${ }_{3}^{2}$ Farmacêtica. Faculdade de Farmácial UFMG - Belo Horizonte-MG.

Universidade Federal de Minas Gerais - Belo Horizonte-MG.

Correspondência: Luci Maria Sant'Ana Dusse

Faculdade de Farmácia - Universidade Federal de Minas Gerais

Av. Antônio Carlos, 6627, Sala 4104 - B3 - Campus Pampulha

31270-901 - Belo Horizonte-MG - Brasil

Tel.: (55 31) 3409-6880 / Fax.: (55 31) 3409-6985

E-mail:lucim@farmacia.ufmg.br

Doi: 10.1590/S1516-84842010005000043
}

${ }^{3}$ Farmacêuticos. Professores da Disciplina de Hematologia Clínica - Faculdade de Farmácia/UFMG - Belo Horizonte-MG. 
microangiopática e sintomas que incluem fraqueza e adinamia, trombocitopenia acentuada, com aparecimento de petéquias e equimoses, febre e sintomas decorrentes da isquemia dos órgãos acometidos. ${ }^{2}$

O comprometimento funcional dos órgãos está diretamente relacionado ao grau de isquemia. Se os trombos ocluírem a microcirculação do cérebro ou dos rins, as consequências podem ser muito graves. No cérebro pode variar de uma simples dor de cabeça ao coma. Nos rins podem acarretar disfunção renal com proteinúria e hematúria. De modo geral, o acometimento renal grave com anúria e uremia excessiva não são típicos de PTT, o que é importante para distingui-la da síndrome hemolítico-urêmica (SHU). A SHU é uma doença também caracterizada por anemia hemolítica microangiopática e trombocitopenia, mas com predominância do quadro renal, que evolui para insuficiência renal aguda. A SHU diferencia-se da PTT por acometer, com frequência, crianças menores de 3 anos de idade, de ambos os sexos, e estar associada à ocorrência de infecções, principalmente por Escherichia coli e Shigella $s p$. Estas bactérias são produtoras de toxinas que se ligam às células endoteliais dos rins e comprometem a função dessas células. ${ }^{2,3}$

Além da SHU, a PTT deve ser diferenciada de outras condições clínicas como anemia hemolítica autoimune, síndrome de Evans, pré-eclâmpsia, infecção pelo vírus da AIDS (HIV), sepse, coagulação intravascular disseminada, endocardites e vasculites secundárias a colagenoses para a intervenção terapêutica adequada. Cerca de metade dos pacientes com PTT relata a ocorrência de uma virose dias antes dos primeiros sintomas da doença. ${ }^{2,4}$

\section{Fator von Willebrand (FvW) e ADAMTS13}

O FvW é uma glicoproteína multimérica composta por subunidades idênticas de $270 \mathrm{kD}$ cada uma, contendo 2.050 aminoácidos. Apenas as células endoteliais e os megacariócitos sintetizam e estocam $\mathrm{FvW}$, e este tem uma vida média de vinte horas. A síntese deste fator ocorre inicialmente no retículo endoplasmático, onde há a formação de pré-dímeros do FvW ligados entre si na porção carboxi terminal por pontes dissulfeto. Estes pré-dímeros migram para o complexo de Golgi, onde outra ponte dissulfeto se forma na porção aminoterminal resultando nos dímeros. Estes dímeros se unem posteriormente, resultando em grandes multímeros ( $\mathrm{gmFvW}$ ), que são armazenados nas células endoteliais (nos corpos de Weibel-Palade) e nas plaquetas. Os gmFvW não são encontrados no plasma de indivíduos normais, sendo sua liberação limitada aos locais de lesão endotelial., ${ }^{5,6}$ Alguns estímulos, como dado pela histamina, toxina Shiga, fator de necrose tumoral $\alpha$ (TNF- $\alpha$ ), interleucina-8 (IL-8) e interleucina-6 (IL-6) promovem a liberação dos gmFvW das células endoteliais para o plasma. O papel fisiológico do FvW inclui a adesão das plaquetas ao endotélio lesado e a participação no processo de agregação entre as plaquetas para formar o trombo plaquetário. Além disso, constitui a proteína carreadora do fator VIII da coagulação, aumentando consideravelmente a meia-vida plasmática desse fator. ${ }^{6}$

Em condições fisiológicas, tão logo os grandes multímeros do fvW são liberados da célula endotelial, esses são clivados e removidos da circulação pela enzima ADAMTS13. ${ }^{7}$ $\mathrm{O}$ termo ADAMTS corresponde à sigla de $A$ Desintegrin And Metalloprotease with eight ThromboSpondin-1-like e o número13 porque essa é a $13^{\text {a }}$ enzima de uma família de 19 enzimas. ${ }^{6,8}$

Juntamente com o FvW, moléculas de selectina P são produzidas pelos corpos de Weibel-Palade nas células endoteliais e secretadas para a membrana dessas células, fixam na porção transmembranar, onde os monômeros de FvW vão enfileirando-se, formando os gmFVW. A molécula de ADAMTS13 liga-se, através da porção carboxi terminal, ao gmFvW, expondo o domínio A2, clivando-o em subunidades monoméricas.

$\mathrm{O} F v W$ é o único substrato conhecido desta enzima e representa apenas $0,02 \%$ das proteínas plasmáticas. ${ }^{8}$

As plaquetas não aderem às pequenas formas de $\mathrm{FvW}$ que circulam após a clivagem dos gmFvW, talvez porque a porção ligante GPIb desse fator para a plaqueta não esteja exposta nestas pequenas formas. ${ }^{5,6}$

A Figura 1 ilustra o mecanismo de secreção e ação da ADAMTS13 em condições normais e na PTT.

\section{Fisiopatologia da PTT}

A fisiopatologia da PTT foi elucidada em 1982 por Joel Moake $e t$ al. ${ }^{5}$ ao observarem que o plasma de um paciente com PTT recorrente continha multímeros de fator von Willebrand $(\mathrm{FvW})$ de alto peso molecular. Estes pesquisadores propuseram que, na PTT, as células endoteliais eram estimuladas de forma anormal a liberar os gmFvW e havia deficiência de uma protease de clivagem desses multímeros. Dessa forma, os gmFvW no plasma promoveriam a agregação intravascular das plaquetas, resultando em trombose microvascular e hemólise mecânica, particularmente em condições de fluxo sanguíneo caracterizado por alto shear stress, como na microcirculação. ${ }^{1,5,9-12}$

No entanto, foi somente em 1996 que Furlan et al. ${ }^{13} \mathrm{e}$ Tsai e $\operatorname{Lian}^{4}$ isolaram uma protease e verificaram que essa era capaz de clivar a ligação peptídica entre a tirosina na posição 1605 e a metionina na posição 1606 no domínio central A2 do FvW. Em um estudo de coorte retrospectivo, estes pesquisadores observaram que os pacientes clinicamente diagnosticados com PTT tinham deficiência desta protease. ${ }^{4,13} \mathrm{Em}$ 2001, Zheng et al. ${ }^{14}$ confirmaram que esta era um novo membro da família das metaloproteases ADAMTS e denominaram-na de ADAMTS13.

A deficiência qualitativa ou quantitativa de ADAMTS13, resultante da mutação do gene responsável 


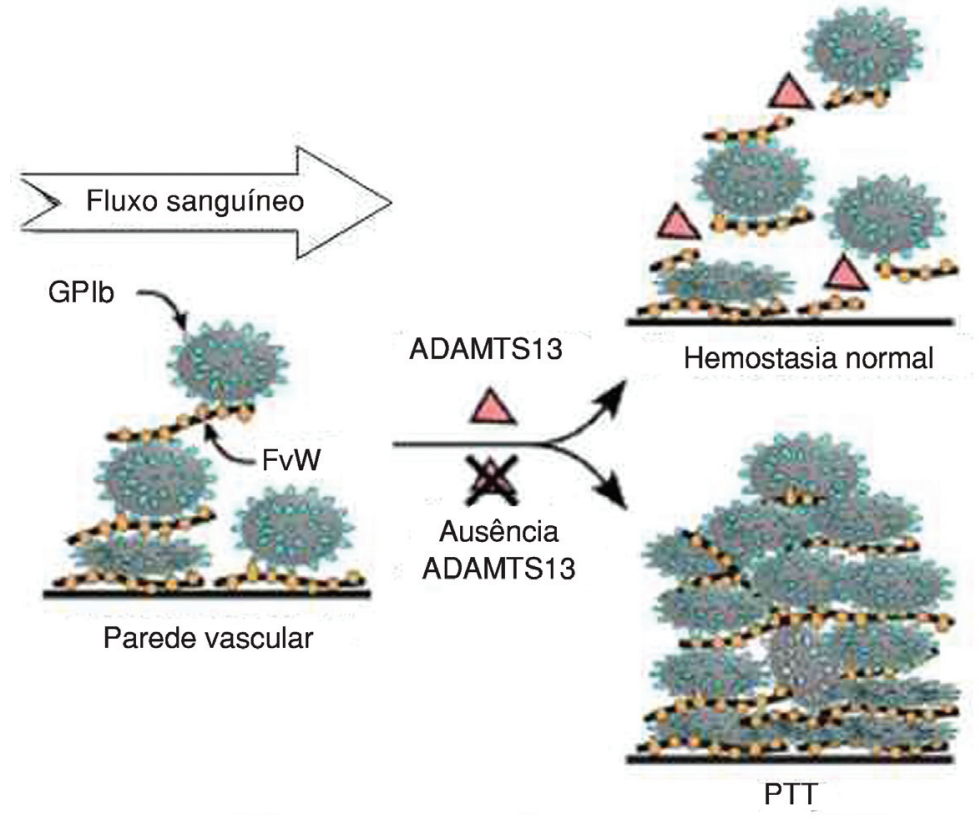

Figura 1. PTT: Púrpura trombocitopênica trombótica

Mecanismo de ação ADAMTS13 em condições normais e formação de agregado plaquetário na ausência desta enzima

por sua síntese ou pela presença de autoanticorpos inibitórios, doenças hepáticas (a síntese de ADAMTS13 ocorre no fígado) ou por outras etiologias, resultam no acúmulo de grandes multímeros de FvW no plasma. $\mathrm{Na}$ ausência ou deficiência desta enzima, as plaquetas circulantes aderem-se às fileiras de gmFVW ancoradas nas selectinas $\mathrm{P}$, através dos receptores GPIb do FvW, e agregam-se resultando na formação de trombos microvasculares (constituídos basicamente de plaquetas e de gmFvW), seguido de hemólise e consumo de plaquetas. ${ }^{6,8}$

\section{Púrpura trombocitopênica trombótica congênita ou hereditária}

A PTT congênita tem curso crônico e é caracterizada por episódios recorrentes de trombocitopenia, com ou sem isquemia de órgãos. Nesta doença tem sido identificada ausência ou redução expressiva na atividade da ADAMTS13, o que propicia a presença de gmFvW em concentrações elevadas na circulação na fase inicial da doença. ${ }^{15} \mathrm{~A}$ alteração da atividade da ADAMTS13 está geralmente relacionada a uma mutação no gene desta enzima (localizada no cromossomo 9q34) e é uma condição autossômica recessiva. Com a evolução do quadro os gmFvW e até os de tamanho normal tendem a desaparecer da circulação e a trombocitopenia acentua-se ainda mais. Tanto as plaquetas quanto os multímeros de FvW são consumidos nos trombos hialinos característicos da enfermidade. A presença dos gmFvW no sangue de pacientes que sobreviveram à PTT congênita constitui uma evidência forte de que esses terão recorrência da doença. A recorrência pode ocorrer em intervalos irregulares ou regulares, como acontece geralmente com indivíduos que tiveram o primeiro episódio na infância. ${ }^{15-18}$

\section{Púrpura trombocitopênica trombótica adquirida}

As formas adquiridas da PTT são classificadas em formas imunomediadas (presença de autoanticorpos anti- ADAMTS13) e as formas secundárias (liberação de grande quantidade de gmFvW pelas células endoteliais estimuladas, excedendo a capacidade de degradação destes, devido à presença de níveis reduzidos de ADAMTS13). ${ }^{19-21}$

As condições fisiológicas ou patológicas que estão mais relacionadas às formas imunomediadas, as quais são frequentemente associadas à deficiência grave de ADAMTS13 (níveis menores que $10 \%$ do normal) incluem a gravidez, infecções, doenças autoimunes e o uso de drogas, tais como ticlopidina e clopidogrel..$^{22}$ Outras condições clínicas concomitantes associadas à PTT adquirida incluem os processos cancerígenos, transplantes de órgãos sólidos ou de células-tronco, pré-eclâmpsia, uso de drogas (ciclosporina, mitomicina e $\alpha$-interferon), dentre outras. Nestes casos, a deficiência de ADAMTS13, em geral, não é muito acentuada. Na maioria dos casos de PTT adquirida, ocorre um único episódio agudo esporádico. No entanto, há formas crônicas recorrentes (20\%-30\% dos casos), as quais têm um fator genético envolvido ou estão associadas à produção e/ou persistência de autoanticorpos anti-ADAMTS13. ${ }^{23}$

\section{Diagnóstico laboratorial da PTT}

O diagnóstico da PTT, em geral, é feito por exclusão de outras possíveis causas da trombocitopenia. O diagnóstico precoce da doença é de fundamental importância, pois o tratamento deve ser instituído prontamente de forma a diminuir a morbidade, evitar procedimentos invasivos, como a esplenectomia, e garantir uma melhor qualidade de vida aos portadores dessa doença.

O laboratório tem um papel fundamental na identificação e relato de alterações, especialmente na série eritrocítica, dentre outros, que possam fornecer subsídios para o correto raciocínio diagnóstico. A hemólise na PTT, de modo geral, é branda, com valores de hemoglobina de aproximadamente $10,0 \mathrm{~g} / \mathrm{dl}$ no momento do diagnóstico. A análise minuciosa do filme sanguíneo é fundamental para o diagnóstico da doença. No filme são observados sinais de hemólise, como a presença de hemácias policromatófilas, microesferócitos, esquizócitos e queratócitos (características 
da anemia hemolítica microangiopática), eritroblastos, hemácias com pontilhado basófilo, além de trombocitopenia intensa, confirmada pela contagem de plaquetas. Outras evidências laboratoriais de hemólise intravascular incluem redução de haptoglobina, hemoglobinúria, hemossiderinúria, aumento da bilirrubina direta e da atividade de desidrogenase lática humana (DLH). A análise do aspirado da medula óssea revela aumento dos precursores da série vermelha e número de megacariócitos normal ou discretamente aumentado. Os exames laboratoriais que investigam os fatores da coagulação, tempo de tromboplastina parcial ativada, tempo de protrombina, fibrinogênio e degradação da fibrina estão normais, o que é importante para diferenciar PTT de coagulação intravascular disseminada (CID). ${ }^{3,24}$

A avaliação laboratorial da ADAMTS13 parece constituir um avanço para o diagnóstico precoce da PTT. Já estão disponíveis no mercado internacional conjuntos diagnósticos para a determinação dos níveis de antígenos da ADAMTS13, da sua atividade e dos anticorpos antiADAMTS13..$^{25-27}$ No entanto, a interpretação dos resultados exige cautela e um conhecimento do princípio do método, bem como das etapas das reações envolvidas. A determinação dos níveis de antígenos desta enzima é feita por enzima imunoensaio (ELISA), mesmo utilizando pequenos peptídeos (FRET-VWF73), ao invés dos multímeros FvW, não assegura o total controle da variabilidade inter e intraensaio. Os testes para a determinação da atividade de ADAMTS13 baseiam-se na clivagem de multímeros do FvW pelaADAMTS13 e na detecção posterior do FvW clivado. ${ }^{28,29}$ Neste teste, a atividade da ADAMTS13 é avaliada em condições estáticas e não reflete as condições fisiológicas do fluxo sanguíneo. Um teste sob fluxo, na tentativa de mimetizar o que ocorre in vivo foi proposto, mas não foi ainda validado. ${ }^{5}$ A maioria dos testes propostos para detecção de anticorpos anti-ADAMTS13 faz a titulação dos anticorpos in vitro, utilizando uma mistura de plasma do paciente e plasma normal inativados a $37^{\circ} \mathrm{C} .{ }^{4,13}$

Fey et al. ${ }^{30}$ compararam os resultados dos níveis de antígenos e da atividade da ADAMTS13 em várias condições fisiológicas e patológicas, como recém-nascidos, gestantes, mulheres em uso de contraceptivo oral, pacientes com cirrose hepática, inflamação intestinal e que fizeram cirurgia cardíaca. Os resultaram mostraram que os níveis de antígeno ADAMTS13 tiveram uma menor variabilidade que a avaliação da atividade da enzima nos diferentes grupos estudados.

A avaliação laboratorial da ADAMTS13 na PTT foi feita pela primeira vez por Furlan et al., ${ }^{13}$ pela determinação da clivagem do FvW em 30 pacientes com PTT aguda. Estes pesquisadores encontraram uma deficiência grave desta enzima em 26 dos casos (86\%). Tsai et al. ${ }^{4}$ também relataram uma deficiência grave de clivagem do FvW em todos os 37 pacientes com PTT avaliados. Veyradier et al. ${ }^{31}$ avaliaram 66 pacientes com PTT e encontraram deficiência da clivagem do
FvW em 59 casos (89\%), dos quais 47 tiveram uma redução drástica $(71 \%)$ desse fator. Mori et al. ${ }^{32}$ também verificaram deficiência importante da clivagem do FvW em todos os 18 pacientes avaliados.

Por outro lado, outros pesquisadores não encontraram uma associação importante da PTT e deficiência da atividade da ADAMTS13. Vesely et al..$^{33}$ relataram que apenas 33\% dos pacientes com PTT tiveram redução importante da atividade desta enzima (16 pacientes em 48). Coppo et $a l .{ }^{34}$ relataram que a deficiência grave da atividade da ADAMTS13 foi verificada em somente $67 \%$ dos 46 pacientes avaliados. Peyvandi et al..$^{35}$ e Zheng et al..$^{36}$ relataram deficiência grave da atividade da enzima em apenas 48 dos 100 pacientes avaliados. Paradoxalmente, uma deficiência da atividade da ADAMTS13 tem sido relatada em outros casos de microangiopatias trombóticas distintos da PTT. ${ }^{37-39}$ Considerando o exposto, é possível inferir que tanto a dificuldade diagnóstica da etiologia da microangiopatia trombótica, como discrepâncias na metodologia de avaliação da atividade da ADAMTS13 possam explicar estas divergências.

A distinção da PTT de outras condições clínicas é essencial. A avaliação da função renal é importante para diferenciar a PTT e a SHU. Na PTT o comprometimento da função renal é moderado, ao contrário do que se observa na SHU, quando o paciente apresenta insuficiência renal franca com elevação da creatinina e ureia que rapidamente atingem níveis que exigem diálise. Além disso, na SHU não se observa deficiência da ADAMTS13 ou inibição dessa por autoanticorpos. Os testes que investigam fatores da coagulação são geralmente normais nas duas condições. ${ }^{24}$

O diagnóstico da PTT muitas vezes constitui um desafio, especialmente quando ocorre microangiopatia trombótica, em associação com outras doenças autoimunes, neoplasias, infecção pelo HIV, transplante de medula, após transfusão sanguínea, durante a gravidez e após uso de alguns medicamentos.

\section{Tratamento}

Uma vez diagnosticada a PTT de qualquer etiologia, o tratamento mais eficaz consiste na troca do plasma, que consiste na plasmaférese (visando retirar os gmFvW da circulação) e infusão de plasma fresco congelado (PFC) ou de sobrenadante do criopreciptado (que contém a ADAMTS13). Este tratamento propicia uma melhora significativa do quadro clínico do paciente em poucas horas. Quando a plasmaférese não está disponível, o paciente deve iniciar a terapia com corticosteroides objetivando a redução da produção de anticorpos antiplaquetários. ${ }^{40}$

A troca do plasma de maneira empírica está preconizada como tratamento primário para PTT idiopática e reduz a mortalidade aguda de mais de $90 \%$ para menos de $20 \%$. Os critérios para selecionar estes pacientes incluem anemia 
hemolítica microangiopática, trombocitopenia e ausência de outra explicação óbvia para a PTT, como câncer, sepse, coagulação intravascular disseminada, transplante de tecidos, uso de determinadas drogas e diarreia sanguinolenta recente. ${ }^{40}$

Na PTT aguda, a plasmaférese deve ser realizada diariamente, com substituição do plasma do paciente por PFC. A resposta deve ser monitorizada todos os dias pela determinação da hemoglobina, contagem de plaquetas, nível de DLH e de creatinina e a presença de esquizócitos, bem como regressão dos sintomas neurológicos. Habitualmente são necessárias várias sessões de plasmaférese, em média por dez dias. Essas não devem ser interrompidas antes que se estabeleça uma remissão estável, com normalização da contagem de plaquetas e dos níveis de hemoglobina e de DLH, por, pelo menos, três dias consecutivos. A interrupção precoce da plasmaférese causa a recorrência da doença em cerca de $40 \%$ a $50 \%$ dos pacientes.

O uso de corticosteroides em doses elevadas (prednisona $2 \mathrm{mg} / \mathrm{kg} / \mathrm{dia}$ ) é recomendado, juntamente com a substituição do plasma, visando a redução dos títulos dos autoanticorpos. Objetivando a redução da mortalidade e morbidade da PTT, vários protocolos terapêuticos têm sido utilizados incluindo a administração de vincristina, imunoglobulina intravenosa, prostaciclina, ciclosporina, esplenectomia, quimioterapia com diversas drogas e até o transplante de medula óssea. A eficácia destes protocolos de tratamento tem se mostrado variável. O uso de vincristina melhorou a contagem de plaquetas, mas não alterou o nível do anticorpo inibidor. ${ }^{40}$

Embora a plasmaférese, associada ao uso de corticosteroides, constitua o tratamento prioritário da PTT e tenha aumentado significativamente a sobrevida destes pacientes, alguns não apresentam uma boa resposta ao tratamento (PTT refratária). Esta consiste em trombocitopenia persistente (contagem de plaquetas inferior a $150.000 / \mathrm{mm}^{3}$ ) ou elevação do DHL após um total de sete sessões de plasmaférese. $^{40}$

O transplante de células-tronco hematopoéticas, após condicionamento com ciclofosfamida, globulina antitimocítica e prednisona foi realizado em um paciente refratário a outros tratamentos e esse teve boa evolução. Considerando a alta mortalidade dos pacientes refratários aos tratamentos usuais, o transplante de células-tronco poderá constituir uma alternativa eficaz, apesar da dificuldade e custo deste procedimento. A esplenectomia tem sido reservada para pacientes refratários aos tratamentos usuais e parece melhorar a resposta à plasmaférese subsequente, bem como reduzir a taxa de recaídas. ${ }^{8,40}$

A demora para iniciar o tratamento da PTT, a presença de estupor ou coma e elevação da creatinina são preditores de insucesso do tratamento e aumento da mortalidade. Nestes casos, uma abordagem mais agressiva, semelhante à doença refratária, é recomendada. Uma das alternativas propostas é a realização de plasmaférese e reposição da volemia com plasma do crioprecipitado, que não contém os multímeros do FvW.

Outra opção é a intensificação dos procedimentos de plasmaférese, ou seja, dobrar a volemia durante as trocas plasmáticas ou realizar a troca de uma volemia plasmática a cada 12 horas. A intensificação é uma medida empírica e somente deve ser considerada em casos de risco de morte. ${ }^{8,40}$

Nos pacientes refratários ao tratamento, a introdução precoce de rituximabe (anticorpo monoclonal contra CD20, que é expresso em células B humanas, e que rapidamente retira essas células da circulação), associado à plasmaférese com reposição de plasma do crioprecipitado, é eficaz para induzir a remissão completa da doença. ${ }^{8,36}$

Como a PTT é uma doença pouco comum, é difícil conduzir estudos prospectivos adequados para avaliar a eficácia de outras modalidades de tratamento, distintos da plasmaférese e infusão de plasma. Assim, as recomendações são baseadas apenas em estudos retrospectivos com número limitado de pacientes. A mortalidade global chega a 20\%, e quase todos os óbitos ocorrem entre aqueles pacientes refratários à plasmaférese. Quanto mais cedo iniciar a plasmaférese, menor a possibilidade de recaídas. ${ }^{36}$

\begin{abstract}
Thrombotic thrombocytopenic purpura (TTP) starts abruptly and is characterized by diffuse occlusion of microcirculation arterioles and capillaries, leading to ischemia of tissues. Occlusion is caused by microscopic clots primarily composed of platelets and von Willebrand factor (VWF). VWF is a multimeric glycoprotein synthesized exclusively by endothelial cells and megakaryocytes. This factor promotes adhesion of platelets to injured endothelium, participates in the process of platelet aggregation and is the carrier protein of factor VIII in the circulation. In physiological conditions, large VWF multimers are present in endothelial cells and platelets and are not present in plasma. As soon as these large multimers are released from the endothelial cell, they are cleaved and removed from circulation by the ADAMTS13 enzyme. A quantitative or functional deficiency of ADAMTS13 results in the accumulation of large VWF multimers in the plasma and may result in the aggregation of platelets and diffuse occlusion of arterioles and capillaries. Most cases of PTT are associated with ADAMTS13 deficiency. The levels of antigens, activity and antibodies of MTS13 can be evaluated using internationally manufactured kits. The laboratory evaluation of ADAMTS13 appears to be a useful tool for the early diagnosis of PTT. However, interpretation of the results requires caution, as well as knowledge of the principles of the method and the steps of the reactions involved. Rev. Bras. Hematol. Hemoter. 2010;32(2):155-161.
\end{abstract}

Key words: Purpura, thrombotic thrombocytopenic; von Willebrand factor; platelet aggregation. 


\section{Agradecimentos}

\section{A Fapemig e CNPq.}

\section{Referências Bibliográficas}

1. Retornaz F, Durand JM, Poullin P, Lefèvre P, Soubeyrand J. Idiopathic thrombotic thrombocytopenic purpura or Moschowitz syndrome: current physiopathologic and therapeutic perspectives. 2000;21 (9):777-84.

2. Sadler JE. Thrombotic thrombocytopenic purpura: a moving target. Hematology Am Soc Hematol Educ Program. 2006:415-20.

3. Peyvandi F, Lavoretano S, Palla R, Feys HB, Vanhoorelbeke K, Battaglioli T, et al. ADAMTS13 and anti-ADAMTS13 antibodies as markers for recurrence of acquired thrombotic thrombocytopenic purpura during remission. Haematologica. 2008;93 (2):232-9.

4. Tsai HM, Lian EC. Antibodies to von Willebrand factor-cleaving protease in acute thrombotic thrombocytopenic purpura. N Engl J Med. 1998;339(22):1585-94.

5. Moake JL, Rudy CK, Troll JH, Weinstein MJ, Colannino NM, Azocar J, et al. Unusually large plasma factor VIII:von Willebrand factor multimers in chronic relapsing thrombotic thrombocytopenic purpura. N Eng J Med. 1982;307 (23): 1432-5.

6. Anstadt MP, Carwile JM, Guill CK, Conklin LD, Soltero ER, Lucci A, et al. Relapse of thrombotic thrombocytopenic purpura associated with decreased VWF cleaving activity. Am J Med Sci. 2002;323(5):281-4.

7. Ruggeri ZM, Mannucci PM, Lombardi R, Federici AB, Zimmerman TS. Multimeric composition of factor VIII/von Willebrand factor following administration of DDAVP: implications for pathophysiology and therapy of von Willebrand's disease subtypes. Blood. 1982;59(6):1272-8.

8. Sadler JE, Moake JL, Miyata T, George JN. Recent advances in thrombotic thrombocytopenic purpura. Hematology Am Soc Hematol Educ Program. 2004;407-23.

9. Eymin G, Andrade M, Andresen M, Pereira J. Thrombotic thrombocytopenic purpura: experience in 18 cases and literature review. Rev Med Chil. 2008;136(12):1518-27.

10. Kwaan HC, Boggio LN. The clinical spectrum of thrombotic thrombocytopenic purpura. Semin Thromb Hemost. 2005;31 (6): 673-80

11. Bianconcini G, Mazzali F, Candini R, Gobbi F. Thrombotic thrombocytopenic purpura (Moschowitz syndrome). A clinical case and review of the literature. Minerva Med. 1993; 84(9): 473-91

12. Rock GA, Shumak KH, Buskard NA, Blanchette VS, Kelton JG, Nair RC, et al. Comparison of plasma exchange with plasma infusion in the treatment of thrombotic thrombocytopenic purpura. Canadian Apheresis Study Group. N Engl J Med. 1991; 325(6):393-7.

13. Furlan M, Robles R, Galbusera M, Remuzzi G, Kyrle PA, Brenner B, et al. von Willebrand factor-cleaving protease in thrombotic thrombocytopenic purpura and the hemolytic-uremic syndrome. N Engl J Med. 1998;339(22):1578-84.

14. Zheng X, Chung D, Takayama TK, Majerus EM, Sadler JE, Fujikawa K. Structure of von Willebrand factor-cleaving protease (ADAMTS13), a metalloprotease involved in thrombotic thrombocytopenic purpura. J Biol Chem. 2001;276 (44): 41059-63.

15. Tsai HM. Molecular mechanisms in thrombotic thrombocytopenic purpura. Semin Thromb Hemost. 2004;30(5):549-57.
16. Schulman I, Pierce M, Lukens A, Currimbhoy Z. Studies on thrombopoiesis.I. A factor in normal human plasma required for platelet production; chronic thrombocytopenia due to its deficiency. Blood. 1960;16:943-57.

17. Upshaw JD Jr.. Congenital deficiency of a factor in normal plasma that reverses microangiopathic hemolysis and thrombocytopenia. N Engl J Med. 1978;298(24):1350-2.

18. Levy GG, Nichols WC, Lian EC, Foroud T, McClintick JN, McGee $\mathrm{BM}$, et al. Mutations in a member of the ADAMTS gene family cause thrombotic thrombocytopenic purpura. Nature. 2001;413 (6855):488-94.

19. Klaus C, Plaimauer B, Studt JD, Dorner F, Lämmle B, Mannucci PM, et al. Epitope mapping of ADAMTS13 autoantibodies in acquired thrombotic thrombocytopenic purpura. Blood. 2004; 103(12):4514-9.

20. Rieger M, Mannucci PM, Kremer Hovinga JA, Herzog A, Gerstenbauer G, Konetschny C, et al. ADAMTS13 autoantibodies in patients with thrombotic microangiopathies and other immunomediated diseases. Blood. 2005;106(4):1262-7.

21. van der Plas RM, Schiphorst ME, Huizinga EG, Hené RJ, Verdonck $\mathrm{LF}$, Sixma JJ, et al. von Willebrand factor proteolysis is deficient in classic but not in bone marrow transplantation-associated, thrombotic thrombocytopenic purpura. Blood. 1999;93(11): 3798-802.

22. Tsai HM. Current concepts in thrombotic thrombocytopenic purpura. Ann Rev Med. 2006;57:419-36.

23. Franchini M, Mannucci PM. Advantages and limits of ADAMTS13 testing in thrombotic thrombocytopenic purpura. Blood Transfus. 2008;6(3):127-35

24. Lourenço DM. Púrpura trombocitopênica idiopática. In: Marco Antônio Zago, Roberto Passeto Falcão, Ricardo Pasquini. (Org.). Hematologia: Fundamentos e Prática. $1^{\text {a }}$ ed. São Paulo: Editora Atheneu. 2001:771-8.

25. Furlan M, Lammle B. Assays of von Willebrand factor-cleaving protease: a test for diagnosis of familial and acquired thrombotic thrombocytopenic purpura. Semin Thromb Hemost. 2002;28(2): 167-72.

26. Veyradier A, Girma JP. Assays of ADAMTS-13 activity. Semin Hematol. 2004;41(1):41-7.

27. Shelat SG, Ai J, Zheng XL. Molecular biology of ADAMTS13 and diagnostic utility of ADAMTS13 proteolytic activity and inhibitor assays. Semin Thromb Hemost. 2005;31(6):659-72.

28. Kokame K, Nobe Y, Kokubo Y, Okayama A, Miyata T. FRETSVWF73, a fluorogenic substrate for ADAMTS13 assay. Br J Haemost. 2005;129(1):93-100.

29. Groot E, Hulstein JJ, Rison CN, de Groot PG, Fijnheer R. FRETSVWF73: a rapid and predictive tool for thrombotic thrombocytopenic purpura. J Thromb Haemost. 2006;4(3):698-9.

30. Feys HB, Canciani MT, Peyvandi F, Deckmyn H, Vanhoorelbeke K, Mannucci PM. ADAMTS13 activity to antigen ratio in physiological and pathological conditions associated with an increased risk of thrombosis. Br J Haematol. 2007; 138(4):534-40.

31. Veyradier A, Obert B, Houllier A, Meyer D, Girma JP. Specific von Willebrand factor-cleaving protease in thrombotic microangiopathies: a study of 111 cases. Blood. 2001;98(6):1765-72.

32. Mori Y, Wada H, Gabazza EC, Minami N, Nobori T, Shiku H, et al. Predicting response to plasma exchange in patients with thrombotic thrombocytopenic purpura with measurement of vWF-cleaving protease activity. Transfusion. 2002;42(5):572-80.

33. Vesely SK, George JN, Lammle B, Studt JD, Alberio L, El-Harake MA, et al. ADAMTS13 activity in thrombotic thrombocytopenic purpura-hemolytic uremic syndrome: relation to presenting features 
and clinical outcomes in a prospective cohort of 142 patients. Blood. 2003;102(1):60-8.

34. Coppo P, Bengoufa D, Veyradier A, Wolf M, Bussel A, Millot GA, et al. Severe ADAMTS13 deficiency in adult idiopathic thrombotic microangiopathies defines a subset of patients characterized by various autoimmune manifestations, lower platelet count, and mild renal involvement. Medicine. 2004;83(4):233-44.

35. Peyvandi F, Ferrari S, Lavoretano S, Canciani MT, Mannucci PM. von Willebrand factor cleaving protease (ADAMTS-13) and ADAMTS-13 neutralizing autoantibodies in 100 patients with thrombotic thrombocytopenic purpura. Br J Haematol. 2004; 127 (4):433-9.

36. Zheng XL, Kaufman RM, Goodnough LT, Sadler J E. Effect of plasma exchange on plasma ADAMTS13 metalloprotease activity, inhibitor level, and clinical outcome in patients with idiopathic and nonidiopathic thrombotic thrombocytopenic purpura. Blood. 2004;103(11):4043-9.

37. Hovinga JA, Studt JD, Alberio L, Lämmle B. von Willebrand factor-cleaving protease (ADAMTS-13) activity determination in the diagnosis of thrombotic microangiopathies: the Swiss experience. Semin Hematol. 2004;41(1):75-82.

38. Mal H, Veyradier A, Brugière O, Da Silva D, Colombat M, Azoulay $\mathrm{E}$, et al. Thrombotic microangiopathy with acquired deficiency in ADAMTS 13 activity in lung transplant recipients. Transplantation. 2006;81(12):1628-32.

39. Blot E, Decaudin D, Veyradier A, Bardier A, Zagame OL, Pouillart P. Cancer-related thrombotic microangiopathy secondary to von Willebrand factor-cleaving protease deficiency. Thromb Res. 2002; 106(2):127-30

40. Fakhouri F, Vernant JP, Veyradier A, Wolf M, Kaplanski G, Binaut $\mathrm{R}$, et al. Efficiency of curative and prophylactic treatment with rituximab in ADAMTS13-deficient thrombotic thrombocytopenic purpura: a study of 11 cases. Blood. 2005;106(6):1932-7.

Avaliação: Editor e dois revisores externos

Conflito de interesse: sem conflito de interesse

Recebido: 22/05/2009

Aceito: 08/12/2009 